\title{
Beeswax organogels: Influence of gelator concentration and oil type in the gelation process
}

\author{
Artur J. Martins ${ }^{\mathrm{a}}$, Miguel A. Cerqueira ${ }^{\mathrm{a}, \mathrm{b}, *}$, Luiz H. Fasolin $^{\mathrm{c}}$, Rosiane L. Cunha ${ }^{\mathrm{c}}$, António A. Vicente ${ }^{\mathrm{a}}$ \\ a Centre of Biological Engineering, University of Minho, Campus de Gualtar, 4710-057 Braga, Portugal \\ b International Iberian Nanotechnology Laboratory, Av. Mestre José Veiga s/n, 4715-330 Braga, Portugal \\ c Department of Food Engineering, Faculty of Food Engineering, University of Campinas - UNICAMP, CEP: 13083-862 Campinas, SP, Brazil
}

\section{A R T I C L E I N F O}

\section{Article history:}

Received 8 February 2016

Received in revised form 28 March 2016

Accepted 29 March 2016

Available online 2 April 2016

\section{Keywords:}

Oleogel

Wax

Rheology

Small angle X-ray scattering (SAXS)

Crystallinity

Food science

\begin{abstract}
A B S T R A C T
This work focused on how different types of oil phase, MCT (medium chain triglycerides) and LCT (long chain triglycerides), exert influence on the gelation process of beeswax and thus properties of the organogel produced thereof. Organogels were produced at different temperatures and qualitative phase diagrams were constructed to identify and classify the type of structure formed at various compositions. The microstructure of gelator crystals was studied by polarized light microscopy. Melting and crystallization were characterized by differential scanning calorimetry and rheology (flow and small amplitude oscillatory measurements) to understand organogels' behaviour under different mechanical and thermal conditions. Fourier transform infrared spectroscopy (FTIR) analysis was employed for a further understanding of oil-gelator chemical interactions. Results showed that the increase of beeswax concentration led to higher values of storage and loss moduli $\left(G^{\prime}, G^{\prime \prime}\right)$ and complex modulus $\left(G^{*}\right)$ of organogels, which is associated to the strong network formed between the crystalline gelator structure and the oil phase. Crystallization occurred in two steps (well evidenced for higher concentrations of gelator) during temperature decreasing. Thermal analysis showed the occurrence of hysteresis between melting and crystallization. Small angle X-ray scattering (SAXS) analysis allowed a better understanding in terms of how crystal conformations were disposed for each type of organogel. The structuring process supported by medium or long-chain triglycerides oils was an important exploit to apprehend the impact of different carbon chain-size on the gelation process and on gels' properties.
\end{abstract}

(c) 2016 Elsevier Ltd. All rights reserved.

\section{Introduction}

Texture is an important quality parameter in fat rich foods, which becomes critical when considering trans- and saturated fats substitution by healthier ingredients. These unhealthy fats are related to coronary heart disease, high blood lipids content, inflammation, oxidative stress, endothelial dysfunction, high body weight, metabolic syndrome among other harmful health implications (Blake, Co, \& Marangoni, 2014; Rogers, 2009). This makes changing consumers' habits, by sacrificing a pleasurable taste and mouth feel, a very problematic issue (Koh et al., 2008), and has been driving the food industry to add fats into food products in order to improve their organoleptic characteristics, aiming at targeting acceptability by the consumers (Acevedo, Peyronel, \& Marangoni, 2011). As a result, food industry faces a great challenge when it comes to find alternatives for trans and saturated fats substitution in food products. Fat-rich foods are considered one of the most desired types of food by consumers, which is understandable recalling that fats provide flavour, mouth feel

\footnotetext{
* Corresponding author at: International Iberian Nanotechnology Laboratory, Av. Mestre José Veiga s/n, 4715-330 Braga, Portugal.

E-mail addresses: amartins@ceb.uminho.pt (A.J. Martins), miguelribeirocerqueira@gmail.com (M.A. Cerqueira).
}

and overall eating pleasure. These alternatives should have good performance in terms of nutritional value (Duffy et al., 2009), while providing good structuring and sensorial attributes. Interesterification processes where fatty acid esters interact with fatty acids, alcohols or other fatty acid esters to form other esters is one of the currently used processes to structure organic solvents such as edible oils (Nugrahini \& Soerawidjaja, 2015; Zárubová, Filip, Kšandová, Šmidrkal, \& Piska, 2010), but this is done involving chemical modification of molecules, which is less and less appreciated by increasingly informed consumers. Structuring edible oils may also be achieved e.g. forming organogels, which can be the response for such challenge, offering a healthier alternative and a customizable performance (Jang, Bae, Hwang, Lee, \& Lee, 2015) In fact, organogels can be integrated in a class of soft functional materials that have been attracting significant interest in recent years as their characteristics can be tailored by varying the structuring agents and/or the oil used (Behera et al., 2015). Their ability to keep gel-like characteristics while using low amounts of gelators/structurants together with their thermoreversibility (Patel, Schatteman, De Vos, Lesaffer, \& Dewettinck, 2013) open the opportunity for application of such edible systems in food products. These bio-based structures consist of an entangled crystal network which can be produced from several types of gelator networks in an effective way (Dassanayake, Kodali, \& Ueno, 2011; Yılmaz \& Ögüütcü, 2014). 
The gelation process is a consequence of the association of microcrystalline structures that are able to build up a three dimensional network that, if strong enough, will be capable of restraining the oil phase with success, resulting in an organogel (Toro-Vazquez et al., 2007). Beeswax (BW) - like other waxes - is able to act as an edible gelator for different edible oils (Dassanayake, Kodali, Ueno, \& Sato, 2009; Ögüücü, Arifoğlu, \& Yılmaz, 2015; Rocha et al., 2013; Yılmaz, Ögütcü, \& Arifoglu, 2015). BW is a complex mixture of chemical compounds predominantly based in straight-chain monohydric alcohol compounds with carbon chains from C24 to C36 and straight-chain acids with carbon skeletons of up to C36, including some C18 hydroxyl acids that can be esters, diesters and triesters (Yilmaz \& Dagdemir, 2012). Beeswax has been used for applications in food products such as a glazing and coating agent, and can be also applied as a texturizer for chewing gum base and as a carrier for additives, thus rendering BW organogels the adequate properties to become an interesting application for future fat-based foods (Yilmaz, Ogutcu, \& Yuceer, 2015; Yilmaz \& Dagdemir, 2012). Studies also showed the ability of BW organogels to maintain aroma characteristics through time, as volatile compounds were successfully incorporated (Öğ̈ütcü, Yılmaz, \& Güneşer, 2015; Yllmaz, Öğütcü, \& Yüceer, 2015).

In this work, organogels were produced and the effects of increasing beeswax concentrations and changing the type (carbon chain size) of oil phase were evaluated. Qualitative phase diagrams, polarized microscopy and dynamic light scattering (DLS) provided information regarding the temperature influence in gelator aggregation. Differential scanning calorimetry (DSC) was subsequently used to evaluate organogel thermal parameters (i.e. melting and crystallization peak temperature and enthalpy changes). Rheological behaviour of organogels was accessed by flow curves and small amplitude oscillatory measurements under isothermal and non-isothermal conditions aiming the establishment of a relationship between organogel structure and functionality. Small angle X-ray scattering (SAXS) was useful to recognise different crystal structures and to relate those with gel strength.

\section{Materials and methods}

\subsection{Raw materials and organogel production}

Beeswax (BW), medium chain triglycerides (MCT, Neobee) and long chain triglycerides (LCT, HOSO) were kindly offered by PothHille (UK), Stepan Lipid Nutrition (USA) and by Cargill (Brazil), respectively.

Organogels were prepared in $8 \times 2 \mathrm{~cm}$ tubes with screw caps by heating the mixture of oil phase (MCT or LCT) and gelator (BW) at $80{ }^{\circ} \mathrm{C}$ during $30 \mathrm{~min}$ under magnetic stirring. Different concentrations of gelator 8, 4, 2 and $1 \%$ in $10 \mathrm{~g}$ of oil phase, were evaluated. The samples were stored at $25 \pm 2{ }^{\circ} \mathrm{C}$ at least during $24 \mathrm{~h}$ before being analysed.

\subsection{Qualitative phase diagrams}

Phase diagrams were constructed using organogels prepared with beeswax and MCT or LCT oils, with different concentrations of gelator $(1,2,4$ and $8 \%$ of gelator steady base of $10 \mathrm{~g}$ of oil for each organogel formed). The range of storage temperatures tested was from 20 to $50{ }^{\circ} \mathrm{C}$ in intervals of $5{ }^{\circ} \mathrm{C}$ and organogels were left for a period of $24 \mathrm{~h}$ at each temperature. The tubes were then tilted and the self-standing ability of the organogel samples was assessed visually; depending on the appearance of the samples and their behaviour those were described as gel, thick liquid or liquid. Samples that did not flow were considered gels, samples that slowly flowed were considered thick liquids and samples that flowed immediately were considered liquids.

\subsection{Dynamic light scattering}

In order to study the aggregation process of beeswax in the presence of MCT or LCT oils, a Zetasizer Nano ZS (Malvern Instruments, UK) using a Helium-Neon (HeNe) laser-wavelength of $633 \mathrm{~nm}$ and a detector angle of $173^{\circ}$ was used (Amin, Barnett, Pathak, Roberts, \& Sarangapani, 2014). After the oil phase heating step, hot structurant solution was poured in a preheated $\left(80^{\circ} \mathrm{C}\right)$ glass cuvette and measurements were performed by cooling the solution in the cuvette from 80 to $0^{\circ} \mathrm{C}$ stepwise with a cooling rate of $1^{\circ} \mathrm{C} /$ step.

\subsection{Polarized microscopy}

Micrographs of organogels were obtained under a polarized light microscope (Olympus System Microscope model BX51TF, Olympus America Inc., Center Valley, PA, USA) equipped with a digital camera (Olympus EX300, Olympus America Inc., Center Valley, PA, USA). The samples after preparation at $80^{\circ} \mathrm{C}$ were poured directly in the support and conditioned at $25^{\circ} \mathrm{C}$ during $24 \mathrm{~h}$ before being analysed at room temperature. Pictures were taken at a magnification of $80 \times$.

\subsection{Differential scanning calorimetry}

Calorimetric studies were performed in a 2920 Modulated DSC differential scanning calorimeter (TA Instruments, USA). A DSC hermetic pan containing organogel sample was tightly sealed and placed in the DSC cell. An empty pan was used as reference. The samples were analysed at $5{ }^{\circ} \mathrm{C} / \mathrm{min}$ from 10 to $90{ }^{\circ} \mathrm{C}$ in two cooling and heating temperature sweeps, for each sample. The crystallization peak temperature $\left(T_{c}\right)$ was considered to be that corresponding to the maximum heat flow and the melting peak temperature $\left(T_{m}\right)$ was considered to be that corresponding to the minimum heat flow. Melting and crystallization enthalpies were determined from the area of the endothermic and exothermic peak, respectively. Indium and water were used as standards for calibration.

\subsection{Rheological analyses}

Flow and oscillatory rheometry were performed using a Physica MCR301 (Anton Paar, Graz, Austria) rheometer at $25^{\circ} \mathrm{C}$. Stainless steel cone-plate geometry of $50 \mathrm{~mm}$, with an angle of $2^{\circ}$ and truncation of $208 \mu \mathrm{m}$ was used. The control of the equipment was made using software Star Rheoplus US200/32. The organogels were tested after structure formation and then the samples were cut and placed in the rheometer Peltier plate. Flow curves for BW organogels were obtained by a three-shear rate sweeps (up-down-up) program, using a shearrate range between 0 and $300 \mathrm{~s}^{-1}$. Results show the behaviour of the samples without prior shearing at the transient state (S1) and then and at the steady state (S3). Thixotropy of the gels was estimated based on the area between curves S1 and S3 (hysteresis). The powerlaw model (Eq. 1) was used to fit the behaviour of non-Newtonian samples, where $\sigma$ is shear stress $(\mathrm{Pa}), \dot{\gamma}$ is shear rate $\left(\mathrm{s}^{-1}\right), \kappa$ the consistency index $\left(\mathrm{Pa} \cdot \mathrm{s}^{\mathrm{n}}\right)$ and $n$ the flow behaviour index (dimensionless).

$\sigma=\kappa \cdot \gamma^{n}$

The apparent viscosity of non-Newtonian samples was determined at 3, 50 and $100 \mathrm{~s}^{-1}$ since some samples showed structure breakdown at higher shear rates. The viscoelastic properties of the organogels were evaluated by oscillatory measurements, using a frequency sweep between 0.01 and $10 \mathrm{~Hz}$, within the linear viscoelastic domain at $25{ }^{\circ} \mathrm{C}$. Non-isothermal analyses consisting in heating-cooling sweeps, were performed between 10 and $80^{\circ} \mathrm{C}$ at $1{ }^{\circ} \mathrm{C} / \mathrm{min}$ with a fixed frequency of $1 \mathrm{~Hz}$. The sample was first placed at $80^{\circ} \mathrm{C}$ and the changes in the slope of complex viscosity $\left(\eta^{*}\right)$ as a function of temperature were evaluated from the derivation of the data in order to better visualize and estimate the thermal transitions. Gel temperature $\left(T_{g}\right)$ was estimated from the $\mathrm{G}^{\prime}$ onset of the abrupt transition during cooling of nonisothermal rheological measurements. 


\subsection{Small angle $X$-ray scattering}

Small angle X-ray scattering (SAXS) measurements were performed at room temperature using the beamline of the National Synchrotron Light Laboratory (LNLS, Campinas, Brazil). The equipment has an asymmetrically cut and bent silicon ( 1111 ) monochromator that yields a monochromatic ( $\lambda=1.54 \AA$ ) and horizontally focused beam. A position-sensitive X-ray detector and a multichannel analyser were used to record the SAXS intensity, $\mathrm{I}(\mathrm{q})$, as a function of modulus of scattering vector $q=(4 \pi / \lambda) \sin (\theta / 2)$ ( $\theta$ being the scattering angle). Each SAXS pattern corresponds to a data collection time of $100 \mathrm{~s}$. The determination of the crystal organization was done using the Bragg law through the relative positions of the diffraction peaks. The lattice parameter $d$ (lamellar periodicity) of the lamellar structures was obtained from the position $\left(s^{*}\right)$ of the first (and most intense) diffraction peak (for lamellar phases the distance between peaks present the relation $1: 2: 3: 4)$. The type of organization of the crystal and the type of crystal structure was evaluated, being the evaluation performed by the Guinier plot, where through the log-log plot $(I)$ and the determination of the slope at small angles it is possible to determine the type of structure present (e.g. flat or rod-type structure). ATSAS software was used to analysis of SAXS data using PRIMUS. Through software was possible to determine the radius of gyration $\left(R_{g}\right)$ (Konarev, Volkov, Sokolova, Koch, \& Svergun, 2003).

\subsection{Fourier transform infrared (FTIR) spectroscopy analysis}

FTIR spectra of the oil, BW and organogels samples were determined using a Fourier transform infrared spectrometer (ATR-FTIR) (PerkinElmer 16 PC spectrometer, Boston, USA). FTIR measurements were made in the wavenumber range of 400 and $4000 \mathrm{~cm}^{-1}$ using 2 scans (Vlachos et al., 2006).

\section{Results and discussion}

\subsection{Temperature phase diagrams}

After BW organogels production they were stabilised at different temperatures (from 20 to $50{ }^{\circ} \mathrm{C}$ in intervals of $5{ }^{\circ} \mathrm{C}$ ) for a period of
$24 \mathrm{~h}$. For evaluation, the tubes were tilted and the self-standing ability of the organogel samples was assessed visually, depending on the appearance of the samples and their behaviour were described as gel, thick liquid or liquid as observed in Fig. 1. Only at beeswax concentrations of $4 \%$ or higher the organogels were able to self-sustain (i.e. gel behaviour) at $20{ }^{\circ} \mathrm{C}$. Results showed few differences between the organogels produced with MCT and LCT as oil phases; the main differences were observed at $30{ }^{\circ} \mathrm{C}$ where LCT organogel with $4 \%$ of BW presented a gel-like behaviour, while the MCT-based organogel for the same concentration of BW presented a partial displacement and did not flow. With these results we were able to understand which minimum BW concentration was able to produce gel-like organogels and then study aggregation by light scattering. Overall both types of organogels presented a very homogeneous and creamy appearance in line with previous studies (Yılmaz \& Öğütcü, 2014).

\subsection{Dynamic light scattering for aggregation study}

With DLS it was possible to evaluate the aggregation process of BW in the studied oils. Fig. 2 shows the scattered light intensity as a function of temperature during the cooling process for different concentrations of gelator in medium (MCT) and long chain (LCT) triglycerides. It is clear that the light scattered remains relatively steady as temperature decreases, and at a certain point an abrupt increase of the scattered light intensity was observed. This increase is dependent on gelator concentration and means that aggregates are being formed (Sawalha et al., 2013). A critical concentration of wax was required to allow forming a crystal network with capability to entrap the oil phase, thus originating a self-standing organogel (i.e. increase of the measured intensity). The different aggregation temperature of the system observed for the same gelator concentration is indicating a possible influence of the oil phase composition. Oil phase type can be responsible for differences in crystallization kinetics, crystal dispersion and the hardness of organogels (Dassanayake, Kodali, Ueno, \& Sato, 2012). This study allowed us to understand that in the range of temperatures from 30 to $60{ }^{\circ} \mathrm{C}$ the aggregation mechanism was mostly influenced by the BW concentration. Therefore the relationship between concentration and temperature is important for understanding these organogels' behaviour and consequent applications.

A

B

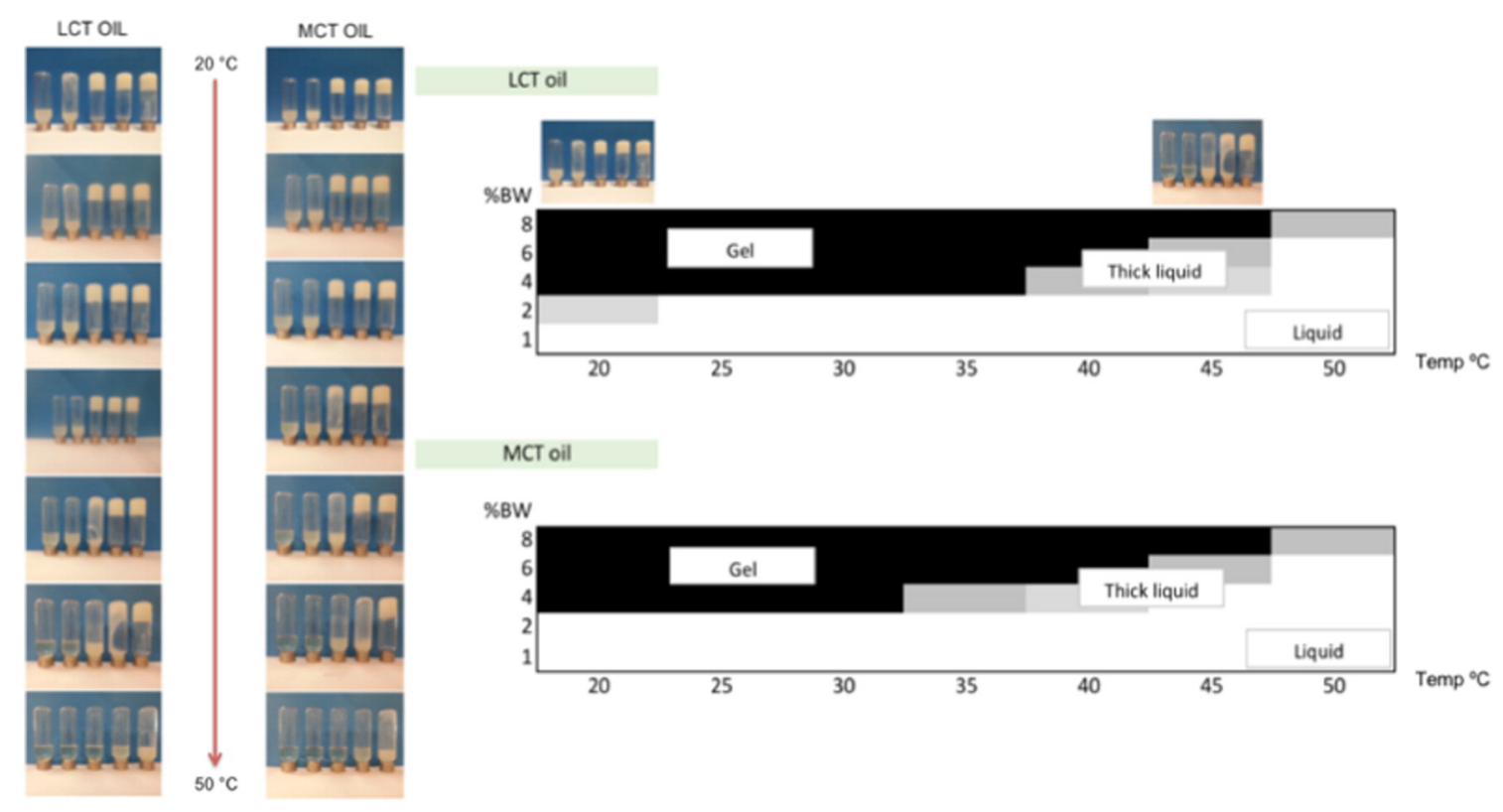

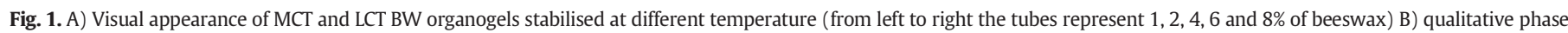
diagrams for MCT and LCT-based organogels with increasing concentrations of beeswax (BW) and different temperature. 


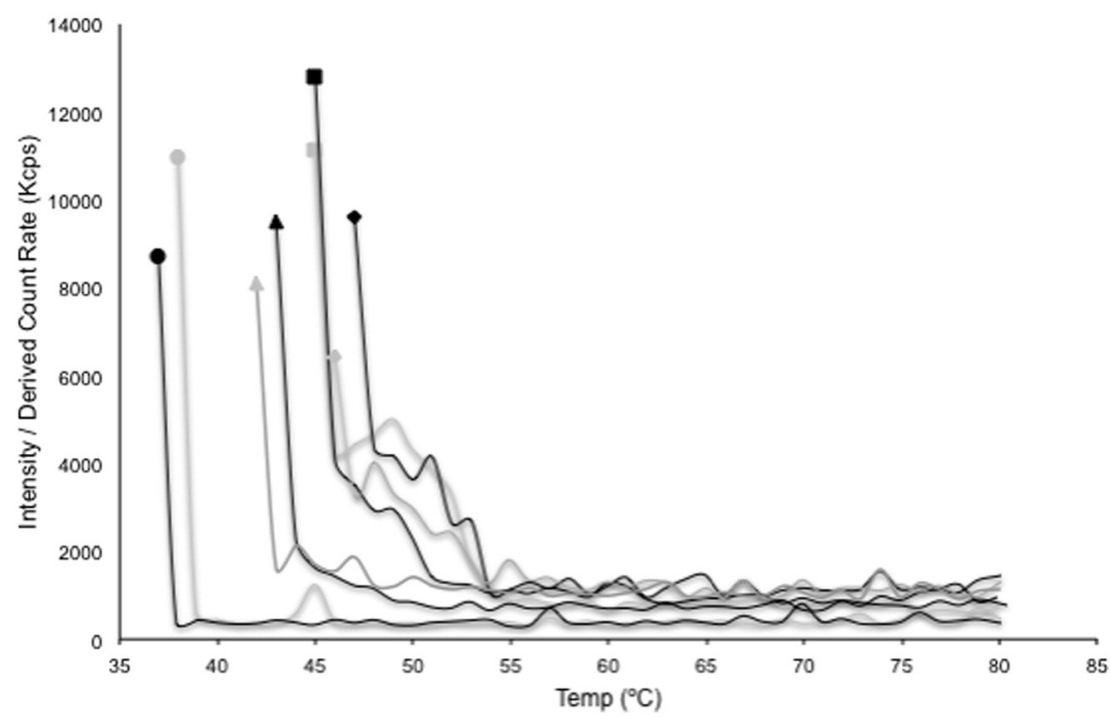

Fig. 2. Scattered light intensity of MCT and LCT-based organogels for $(\bullet) 2,(\mathbf{\Delta}) 4$, ( $\mathbf{\square}) 6$ and $(\bullet) 8 \%$ of BW as a function of temperature (MCT displayed in black and LCT in grey).

\subsection{Polarized microscopy}

Crystal morphology and crystal arrangement was studied for a better understanding of the influence of gelator concentration in organogel formation. The morphology of the crystals was observed under a polarized light microscope. Fig. 3 shows the increase of birefringence for higher concentrations of BW. The crystalline network undergoes significant changes when the concentration of BW is increased from 2 to $4 \%$ for both oil phases (Fig. 3A to B and D to E). The differences are greater when comparing 2 and $4 \%$ BW organogels than when comparing 4 and $8 \%$ BW organogels. At room temperature both types of organogels (MCT- and LCT-based) presented the same characteristics concerning crystal morphology and arrangement.

\subsection{Calorimetric analysis}

Table 1 shows the parameters obtained from DSC analyses. For an appropriate understanding of the meaning of the thermodynamic characteristics for these organogels, it is very important to consider the chemical nature and molecular composition of beeswax and how this gelator is able to interact with the oil phase in both melting and crystallization processes. The increase of crystallization temperature alongside with the increase of gelator concentration in the organogel development stage can be related to the increase of amount of BW concentration leading to the consequently stronger crystal network (as confirmed by the polarized micrographs). Similar thermal behaviour was reported for BW organogels with hazelnut oil (Yılmaz \& Öğ̈utcü, 2014). The predominant
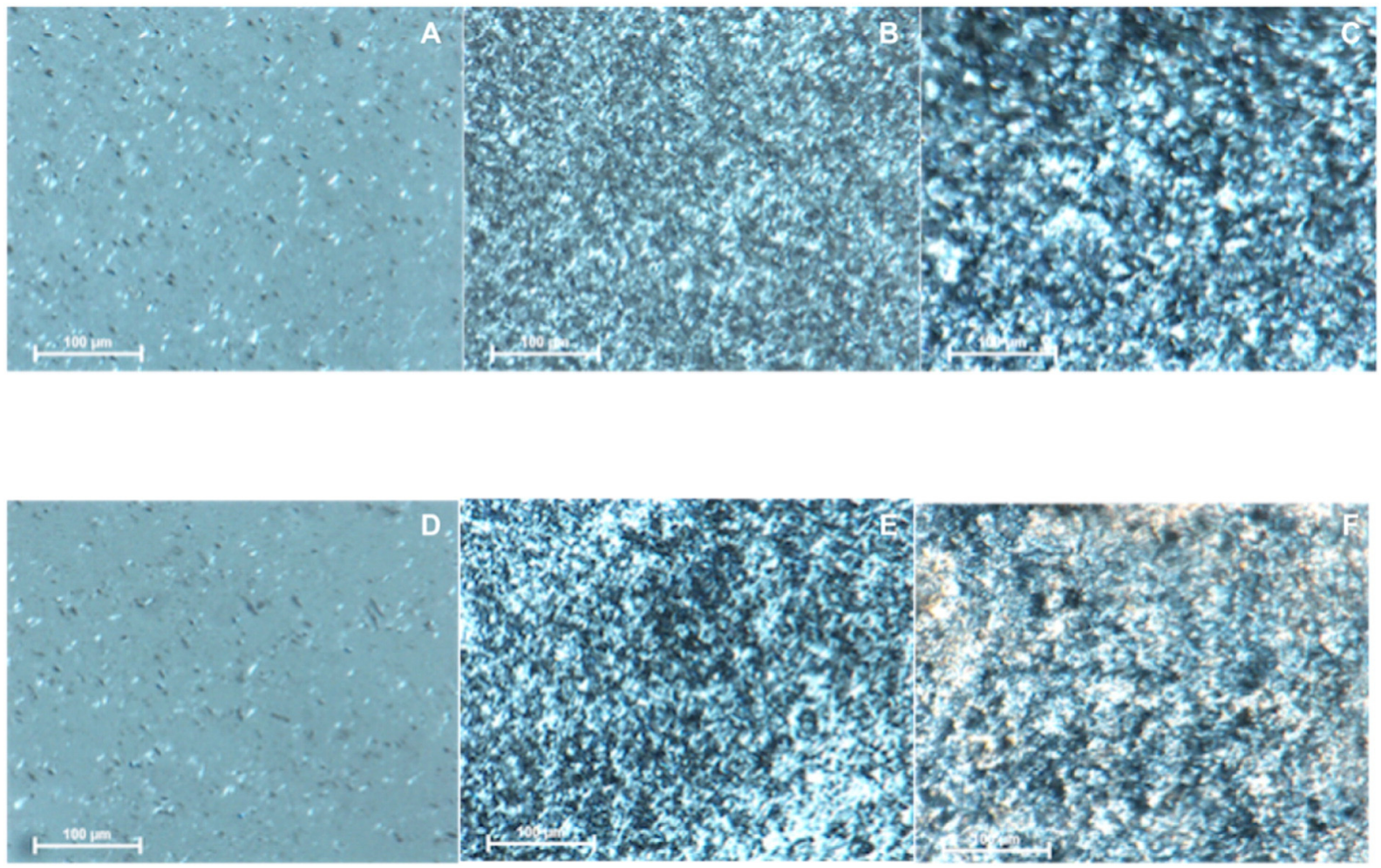

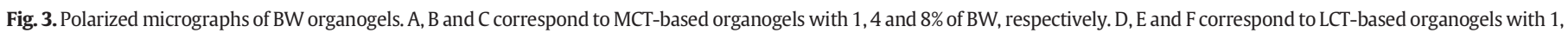
4 and $8 \%$ of BW, respectively. 


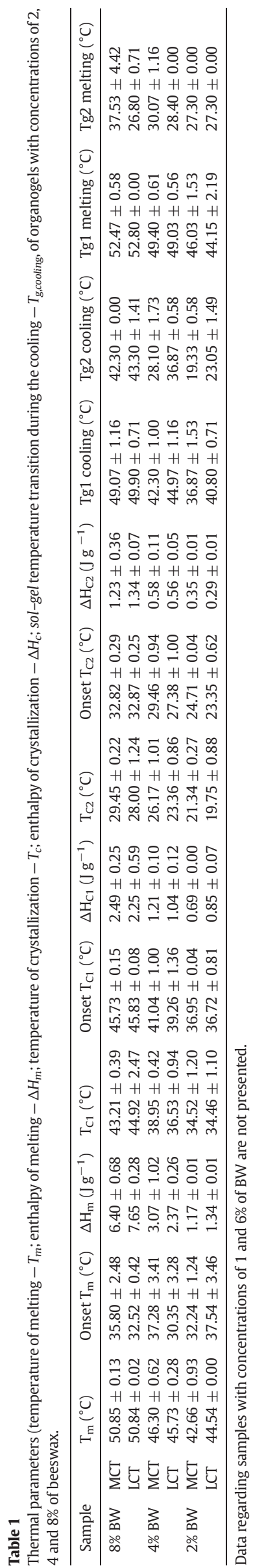

hydrocarbon and linear ester chains should be responsible for the alignment of the saturated chains that will be accountable for the entrapment of the oil phase (Pérez-Monterroza, Márquez-Cardozo, \& Ciro-Velásquez, 2014). Enthalpy changes both for melting and crystallization is well differentiated and related with the gelator concentration. Hysteresis is well observed between $T_{m}$ and $T_{c}$ when the gel-solid transition occurs as temperature decreases. BW crystals were formed from two different stages of crystallization which were identified in the thermogram. The presence of more than a single exothermic peak is a result of the heterogeneous chemical composition of the BW (Blake et al., 2014). This can result from hindered crystal growth, due to the low compatibility between major and minor components, as debated by Hwang et al. This degree of crystalline disorder can be responsible for a crystallization in two different stages, since first a group of BW crystals start to form as the first onset temperature (Onset $T_{C 1}$ ) is reached and then after $T_{C 1}$ a lower amount of energy is needed for a final arrangement of the crystal network to complete the structuring process of the BW organogels.

\subsection{Rheological measurements}

\subsubsection{Non-isothermal}

From the region between 80 and $10{ }^{\circ} \mathrm{C}$ two plateaus and one abrupt transition are observed. Another transition with a smaller slope is noticed (Fig. 4) at temperatures between 10 and in the range between 40 and $50{ }^{\circ} \mathrm{C}$ and correspond to the phase transition of the organogels, which was more pronounced in the MCT organogels. The slope, or abrupt transition, observed in Fig. 4 expresses a rapid structural conversion during cooling/heating from liquid or molten organogel, to the final crystalline state. The gelator concentration (saturation) is responsible for the differences in the onset temperatures and state transition (crystallization/melting stages) in organogels, which was also observed in DSC data (Table 1). The increase of BW concentration leads to higher values of the onset $T_{C 1}$ and consequently $T_{C 1}$ as well. $T_{C 1}$ is in the range $34.46-44.92{ }^{\circ} \mathrm{C}$ and $\mathrm{T}_{\mathrm{g}}$ is in the range $36.87-49.90{ }^{\circ} \mathrm{C}$ for all organogels showing the same behaviour in both measurements. A gradual increase is noticed as more gelator is added to the formulation, leading to a change in the $T_{C 1}$ values. For an increase of $6 \%$ in BW concentration (from 2 to $8 \%$ of BW) a change of approx. $10{ }^{\circ} \mathrm{C}$ in the $T_{C 1}$ values occurs, being this one the highest change when the same comparison is made between 4 and $8 \%$ of BW organogels. Overall, during both heating and cooling stages, the evaluated rheological parameters are higher for LCT organogels.

During the heating phase $G^{\prime}$ and $G^{\prime \prime}$ values showed a more pronounced decrease for organogels using MCT as oil phase; this decrease was more evident between 45 and $55^{\circ} \mathrm{C}$. After that temperature interval, the curves are very alike which is possibly due to the complete melting of gelator network in organogels (for both oil phases). Melting temperature $\left(T_{m}\right)$ values obtained by DSC measurements are also in agreement with the rheological results. The values for $T_{m}$ ranged from $44.54{ }^{\circ} \mathrm{C}$, for $2 \% \mathrm{BW}$ organogels, to $50.85^{\circ} \mathrm{C}$, for $8 \% \mathrm{BW}$ organogels, which correspond to the same range of temperatures that can be identified in the rheological temperature sweeps as the turning point where the organogels turn into a molten state. These sudden transitions took place at different ranges of $G^{\prime}$ and $G^{\prime \prime}$; this change in the rheological parameters is a consequence of varying concentrations of gelator.

Regarding the cooling phase, the slope changes observed for the rheological parameters $G^{\prime}$ and $G^{\prime \prime}$ are a result of the crystallization process that is developed in more than one single stage due to formation of diverse polymorphic crystalline structures (Rocha et al., 2013). During this stage $G^{\prime}$ and $G^{\prime \prime}$ values increase as the temperature decreases, which is a consequence of gelator crystal network assembly and consequent organogel formation. This behaviour suggests that crystallization occurs in two steps; the temperature sweep analysis reveals a sudden increase and then a plateau followed by another slightly increasing curve; this is also verified by DSC analyses, where two distinct crystallization peaks or slopes take place for organogels with BW concentration 

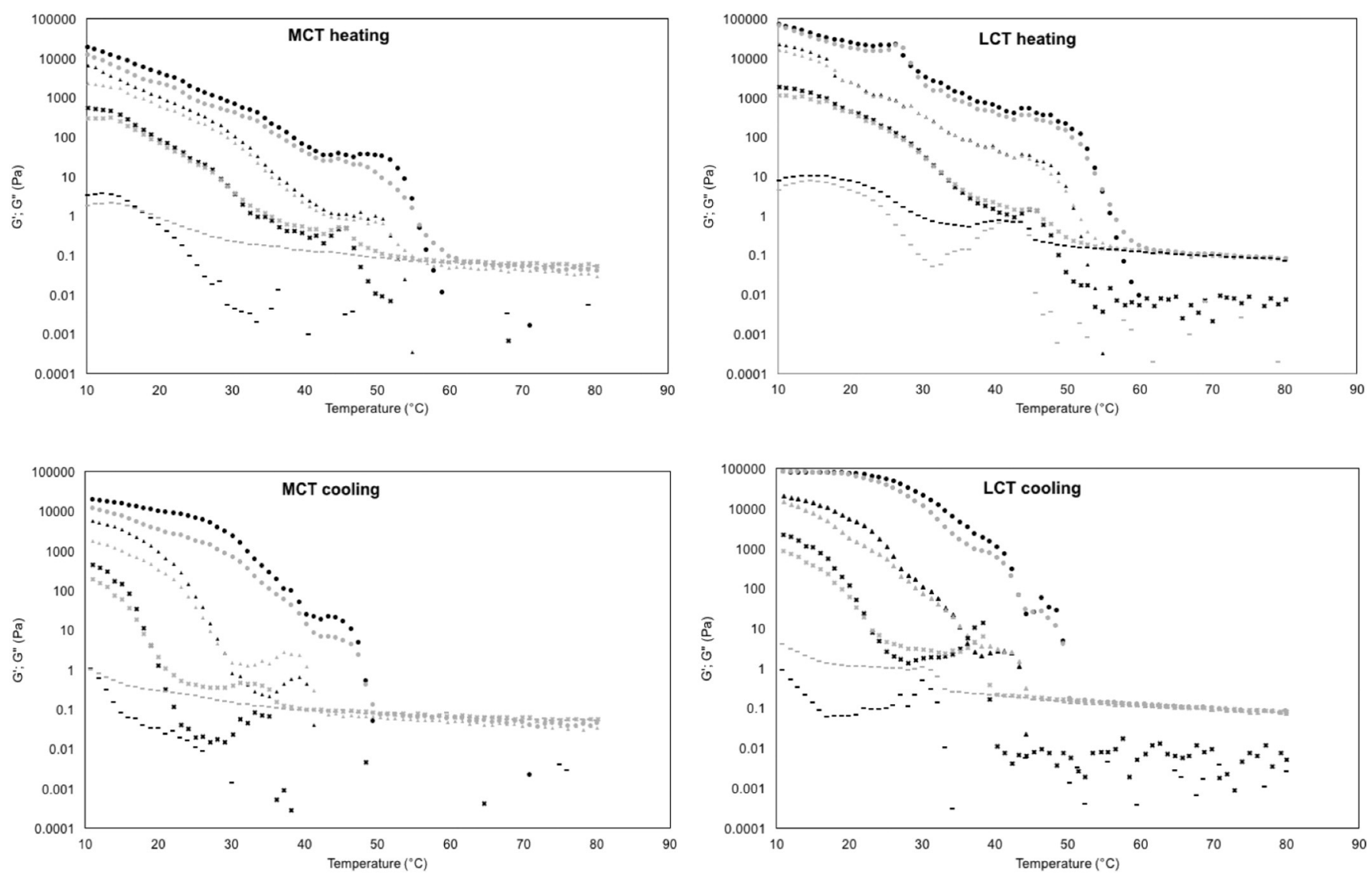

Fig. 4. Temperature sweeps, heating and cooling stages are shown, $(-) 2,(\mathrm{x}) 4,(\boldsymbol{\Delta}) 6$ and $(\bullet) 8 \%$; $G^{\prime}$ displayed in black and $G^{\prime \prime}$ in grey.

above $2 \%$. Results show organogels' behaviour concerning crystal formation and oil-gelator interaction; overall, $T_{m}$ and $T_{c}$ are in the same range of values for MCT and LCT organogels. The resemblance between the rheological temperature sweeps and DSC values, for both types of organogels, shows the relationship between the rheological behaviour and the corresponding thermal characteristics. However, LCT based organogels presented higher values of $G^{\prime}$ and $G^{\prime \prime}$ which could be associated with longer chain oil or lower mobility of gelator and strengthened network of gels formed during crystallization process.

\subsubsection{Isothermal rheology: frequency sweeps and flow curves}

Fig. 5 shows complex modulus $\left(G^{*}\right)$ profiles of organogels after crystallization $\left(25^{\circ} \mathrm{C}\right)$ as a function of the frequency for all the studied concentrations of gelator. A clear increase of organogel strength is observed alongside with increase of BW concentrations, indicating the formation of stronger association between the crystallized gelator structures and the oil phase (reflected in the increase of the $G^{*}$ values), which indicates a more strengthened structure, able to withstand higher stress values before irreversible deformation. The increase of $G^{*}$ values was associated to

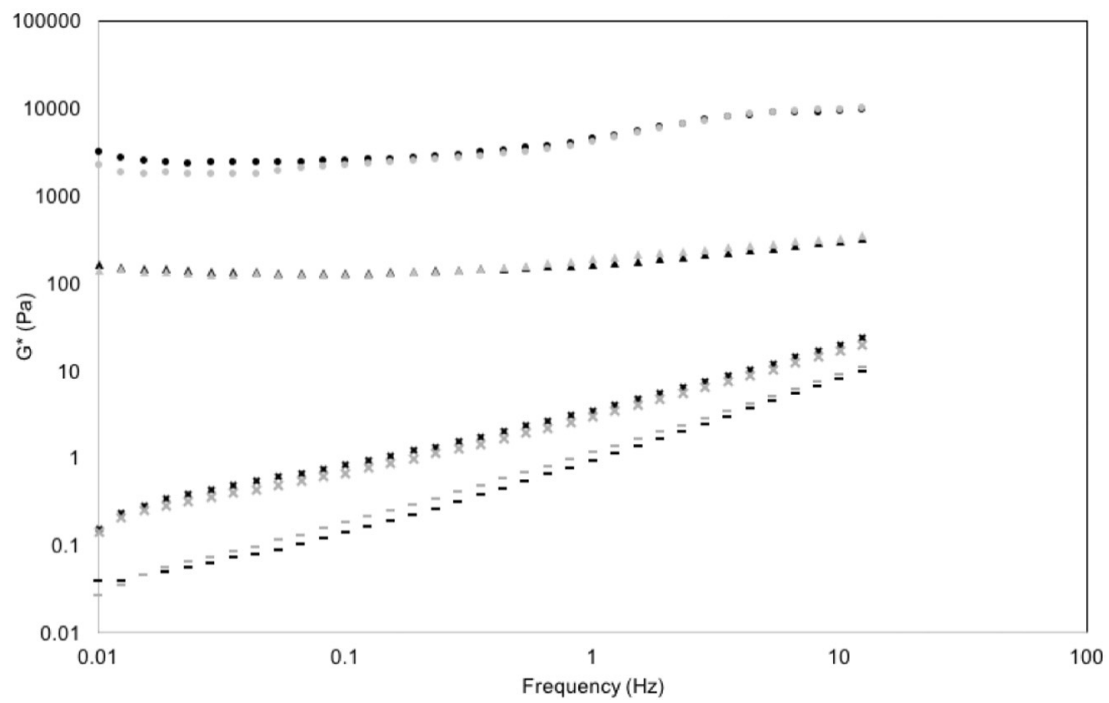

Fig. 5. Complex modulus $\left(G^{*}\right)$ as function of frequency for MCT- and LCT-based BW (-) 1, (x) 2, ( $\left.\mathbf{\Delta}\right) 4$ and $(\bullet) 8 \%$; MCT displayed in black and LCT in grey. 
an increase of the BW's solid volume fraction of the organogels under evaluation (Blake et al., 2014). The independence of frequency (a characteristic of stronger gels) is more evidenced for organogels with higher concentrations of gelator ( $4 \%$ and $8 \%$ of BW). The $G^{*}$ profile expressed for $1 \%$ and $2 \%$ of BW concentration reveals a strong frequency dependence, since the gelator concentration is not enough to create a complex crystal network. The values for MCT- and LCT-based organogels were quite similar.

Fig. 6 shows the stress overshoot observed for the highest concentration of BW organogels ( $8 \%$ ) with MCT or LCT oil registered between shear rate values of 0 and $50 \mathrm{~s}^{-1}$ indicating breakage of a more structured crystalline network with the increase of shear rate. For instance the act of swallowing normally occurs at a shear rate in the range from 5 to $50 \mathrm{~s}^{-1}$ (Cichero \& Murdoch, 2006), in contrast with pipe flow (e.g. blood flow; pumping) which range from 1 to $1000 \mathrm{~s}^{-1}$ (Barnes, Hutton, \& Walters, 1989).

At lower concentration of gelator (4\%), the organogels showed a low structuring behaviour revealed by a small stress overshoot, which can be related to a weaker network (shown in polarized micrographs and thermal analyses). The shear stress values of the flow curves of MCT oil organogels were similar to those found for LCT oil organogels.

Organogels with 4 and $8 \%$ of BW showed thixotropy, being the highest values of hysteresis associated with organogels produced with $8 \%$ of BW because of the more structured crystal network formed by self-assembly (Table 2). Organogels with 1 and $2 \%$ of BW did not present hysteresis or shear time dependence (i.e., non-thixotropic behaviour). Food processing/usage of gels are associated to different shear rate values and the viscosity of Non-Newtonian materials changes according to the shear rate. Therefore, the values of viscosity of the BW organogels with MCT and LCT oil were determined at different shear rate conditions, such as storage or almost quiescent conditions $\left(\eta_{3}\right)$, human act
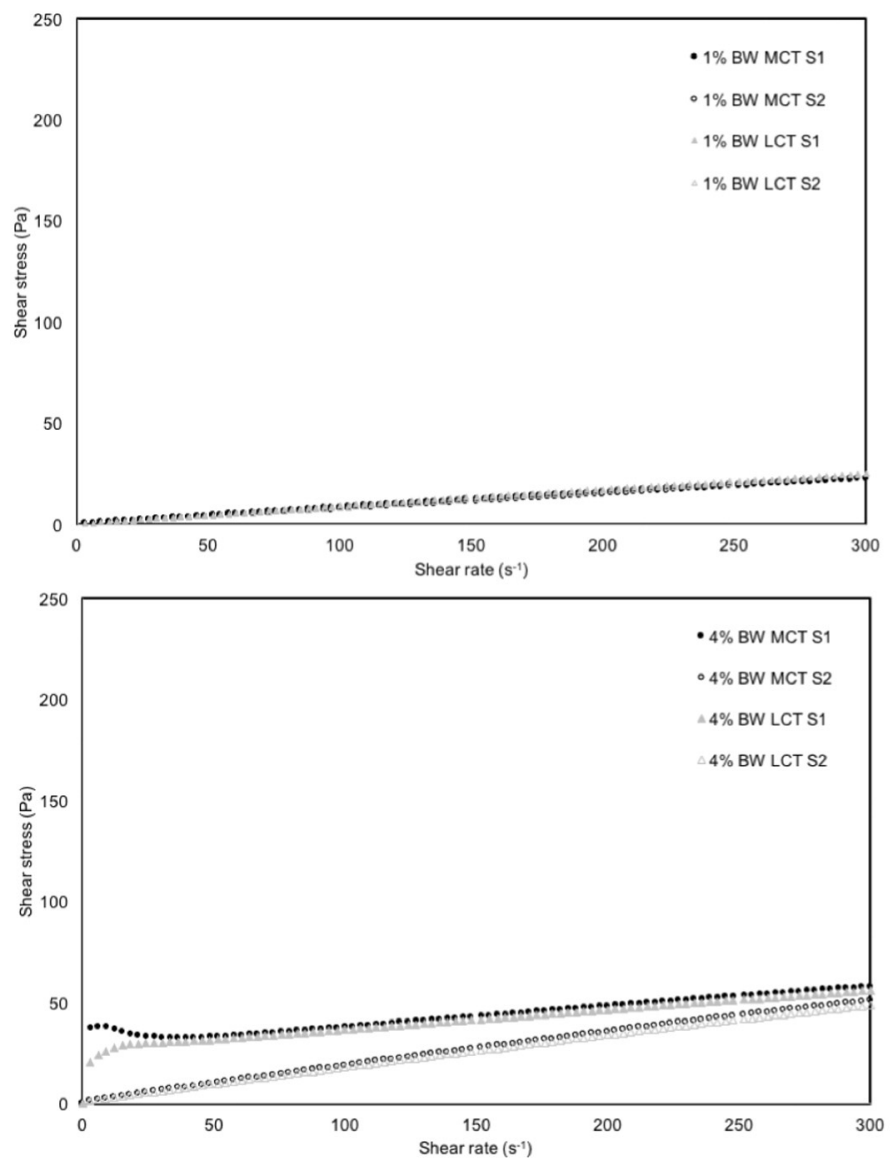

of swallowing $\left(\eta_{50}\right)$ and pipe flow in industrial processing $\left(\eta_{100}\right)$. These are relevant parameters for the development of new products as well as for determining their industrial processability and possible relevance for the consumers. A slight shear-thinning behaviour was observed for $8 \% \mathrm{BW}$ organogels. Regarding $8 \% \mathrm{BW}$ organogels, the viscosity value in steady state $\left(\eta_{3}\right)$ is quite similar to that of chocolate syrup; when ingested $\left(\eta_{50}\right)$ and processed $\left(\eta_{100}\right)$ the value decreases reaching values comparable to a starch solution or sugar solution of $70^{\circ}$ Brix. Organogels with $4 \%$ of BW reveal viscosity values resembling liquid egg solution (under ingestion and industrial processing conditions). Organogels with 2 and 1\% of BW present viscosities in the same range for the three studied parameters since they present a behaviour close to a Newtonian fluid. The $2 \% \mathrm{BW}$ organogels' viscosity value is similar to the one reported for rapeseed oil and $1 \%$ BW organogels present a similar behaviour to that of soybean or olive oil.

\subsection{SAXS}

Fig. 7 shows SAXS spectra regarding each type of oil phase, giving information about crystal structure and the way that these crystals are organized in the bulk organogels. Data concerning LCT-based organogels presented one smaller peak, followed by a sharp one that was tailed by a weaker reflection. Thus, at least three peaks were observed for all gelator concentrations above gelation critical concentration (4\%). For MCT-based organogels the spectra showed a single, small and wide peak that was followed by an even feebler replication. The presence of at least two peaks respecting the relation 1:2 is typical of lamellar structures and the differences between $d$-spacing and $R_{g}$ values can be related to the gel properties. Platelet-like crystals are formed when this type of gelators are employed to structure edible oils (Blake \& Marangoni,
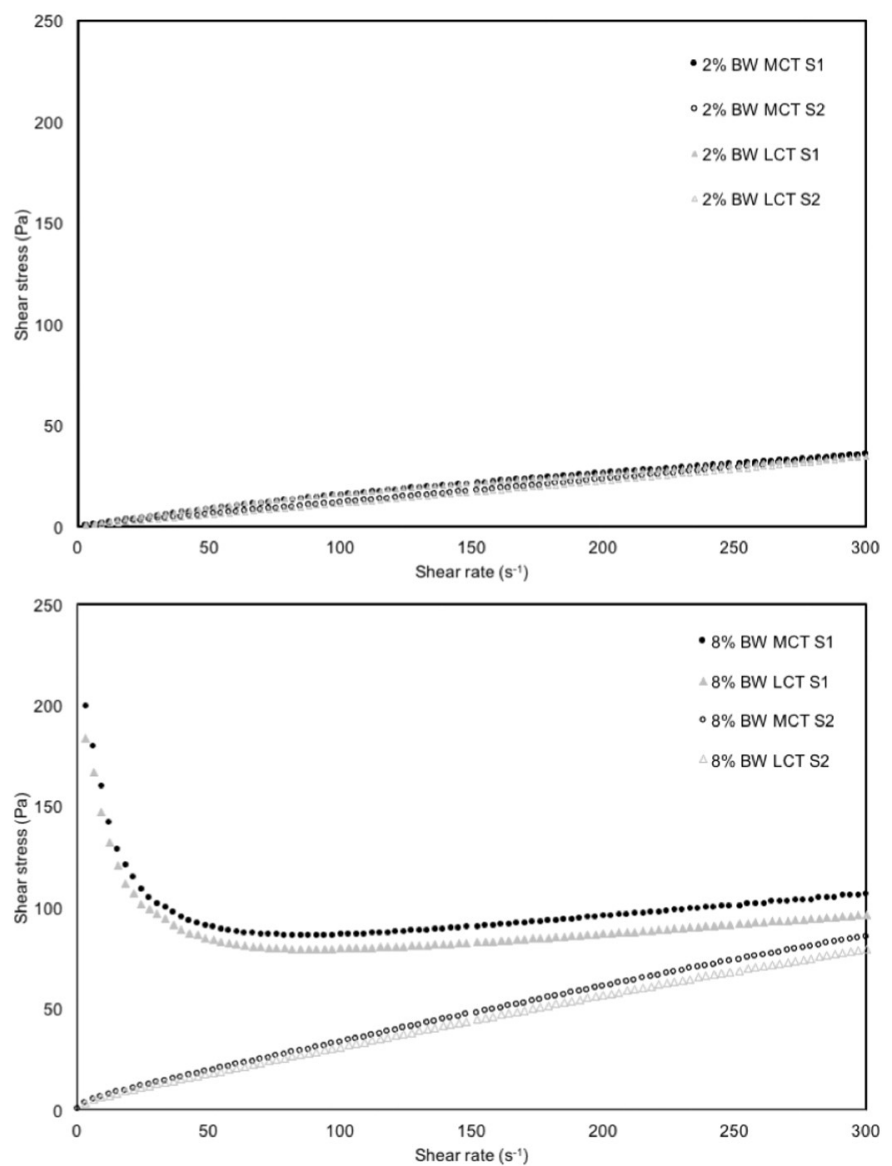

Fig. 6. Flow curves for all concentrations of BW organogels with MCT and LCT. 
Table 2

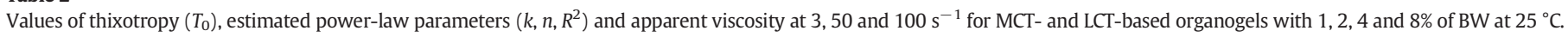

\begin{tabular}{|c|c|c|c|c|c|c|c|c|}
\hline Sample & & $\mathrm{T}_{0}$ & $\mathrm{~K}\left(\mathrm{~Pa} \cdot \mathrm{s}^{\mathrm{n}}\right)$ & $\mathrm{n}$ & $\mathrm{R}^{2}$ & $\eta_{3}(\mathrm{~Pa} \cdot \mathrm{s})$ & $\eta_{50}(\mathrm{~Pa} \cdot \mathrm{s})$ & $\eta_{100}(\mathrm{~Pa} \cdot \mathrm{s})$ \\
\hline \multirow[t]{2}{*}{$8 \%$ BW } & MCT & $2.52 \pm 0.24$ & $0.51 \pm 0.02$ & $0.90 \pm 0.00$ & $0.99 \pm 0.00$ & $1.23 \pm 0.03$ & $0.39 \pm 0.01$ & $0.34 \pm 0.01$ \\
\hline & LCT & $2.64 \pm 0.28$ & $0.45 \pm 0.03$ & $0.90 \pm 0.00$ & $0.99 \pm 0.00$ & $1.16 \pm 0.12$ & $0.36 \pm 0.03$ & $0.31 \pm 0.02$ \\
\hline \multirow[t]{2}{*}{$4 \%$ BW } & MCT & $0.35 \pm 0.05$ & $0.26 \pm 0.02$ & $0.92 \pm 0.00$ & $0.99 \pm 0.00$ & $0.39 \pm 0.03$ & $0.19 \pm 0.01$ & $0.18 \pm 0.01$ \\
\hline & LCT & $0.40 \pm 0.02$ & $0.27 \pm 0.01$ & $0.92 \pm 0.00$ & $0.99 \pm 0.00$ & $0.40 \pm 0.01$ & $0.20 \pm 0.01$ & $0.19 \pm 0.01$ \\
\hline \multirow[t]{2}{*}{$2 \% \mathrm{BW}$} & MCT & $0.00 \pm 0.00$ & $0.14 \pm 0.00$ & $0.96 \pm 0.00$ & $0.99 \pm .00$ & $0.16 \pm 0.00$ & $0.12 \pm 0.00$ & $0.12 \pm 0.00$ \\
\hline & LCT & $0.00 \pm 0.00$ & $0.14 \pm 0.00$ & $0.96 \pm 0.00$ & $0.99 \pm 0.00$ & $0.14 \pm 0.00$ & $0.11 \pm 0.00$ & $0.11 \pm 0.00$ \\
\hline \multirow[t]{2}{*}{$1 \%$ BW } & MCT & $0.00 \pm 0.00$ & $0.11 \pm 0.02$ & $0.96 \pm 0.04$ & $0.99 \pm 0.00$ & $0.09 \pm 0.01$ & $0.08 \pm 0.01$ & $0.08 \pm 0.01$ \\
\hline & LCT & $0.00 \pm 0.00$ & $0.10 \pm 0.02$ & $0.96 \pm 0.03$ & $0.99 \pm 0.00$ & $0.09 \pm 0.00$ & $0.08 \pm 0.00$ & $0.08 \pm 0.00$ \\
\hline
\end{tabular}

2015). These platelets produced by stacking of wax crystals are formed in a lamellar shape, which will be responsible for oil entrapment.

Regarding LCT-based organogels the intensity of the first peak increased as the gelator concentration increased as well; for a concentration of BW of $2 \%$ this first peak was barely noticeable (Fig. 7B). The sharp peak verified for all LCT-based organogels occurred approximately at $q=0.93 \mathrm{~nm}^{-1}$ and the crystalline structures were found to have $d$ spacing values of approximately $67 \AA$ for $2 \%$ of BW, and $93 \AA$ for the remainder $4-8 \%$ of BW. MCT-based organogels presented the highest peak near $q=0.60 \mathrm{~nm}^{-1}$, and $d$-spacing of lamellas of approximately 106 Å for all organogels with 2-6\% BW concentrations. In the later, it was possible to verify the formation of different crystalline organization as the amount of BW is increased, since at $8 \%$ the peak displaced to a lower q-value. $R_{g}$ values for LCT-based organogels were in the range of 29.10 to $33.50 \AA$, which was considerably higher than the values corresponding to MCT-based organogels in the range of 6.33 to $19.70 \AA$ (Fig. 7C). $R_{g}$ analysis reckons the development of thicker lamellar structures for LCT-based organogels. For lower concentrations of gelator, both LCT and MCT-based organogels presented the same lamellar (which is of 1:2:3:4 for lamellar structures) organization, however as gelator concentration was increased, LCT-based organogels developed a different packing structure due to larger complexes of crystal deposition. The increase of concentration of the gelator lead to a different crystalline deposition within the formed lamellas. The lower spacing between lamellas and their larger structure (as confirmed by the higher $R_{g}$ values) in LCT-based organogels can be responsible for the increased values of
$G^{\prime}$ and $G^{\prime \prime}$ verified in the non-isothermal rheology experiments, as shear-induced gelation was responsible for a stronger organogel. The increased intensity of peaks verified for organogels with higher concentrations of gelator indeed demonstrated the successful formation of a more structured self-assembled three-dimensional structure.

\subsection{FTIR}

Fig. 8 presents the superimposed spectra for selected samples of BW, MCT and LCT oil, as well as MCT and LCT organogels. Spectral changes are observed in the $\mathrm{C}-\mathrm{O}$ region $\left(\sim 1746 \mathrm{~cm}^{-1}\right)$, where a widening of the bands is shown, possibly due to heating and the consequent production of saturated aldehyde functional groups (oil phase) or other secondary oxidation products (Vlachos et al., 2006). The addition of BW enhances this effect in the MCT organogel, broadening the band to lower wavenumbers. The combination of heat and BW addition could be the cause for the overlapping of the stretching vibration of the ester carbonyl functional group of triglycerides. Absorbance is observed in the $3005 \mathrm{~cm}^{-1}$ fingerprint region of the spectrum, due to the $\mathrm{C}-\mathrm{H}$ stretching of the cis-double bonds. Typically, MCTs do not show absorbance in this region, while LCTs do, which is clear from Fig. 8. Also in this region, the presence of BW in LCT sample (10\% BW LCT) was confirmed.

Apart from the oil fingerprint region there was no new peak formation, peak disappearance or peak shifts for the conjugation of BW and the different oil phases, which is a clear indication that only physical
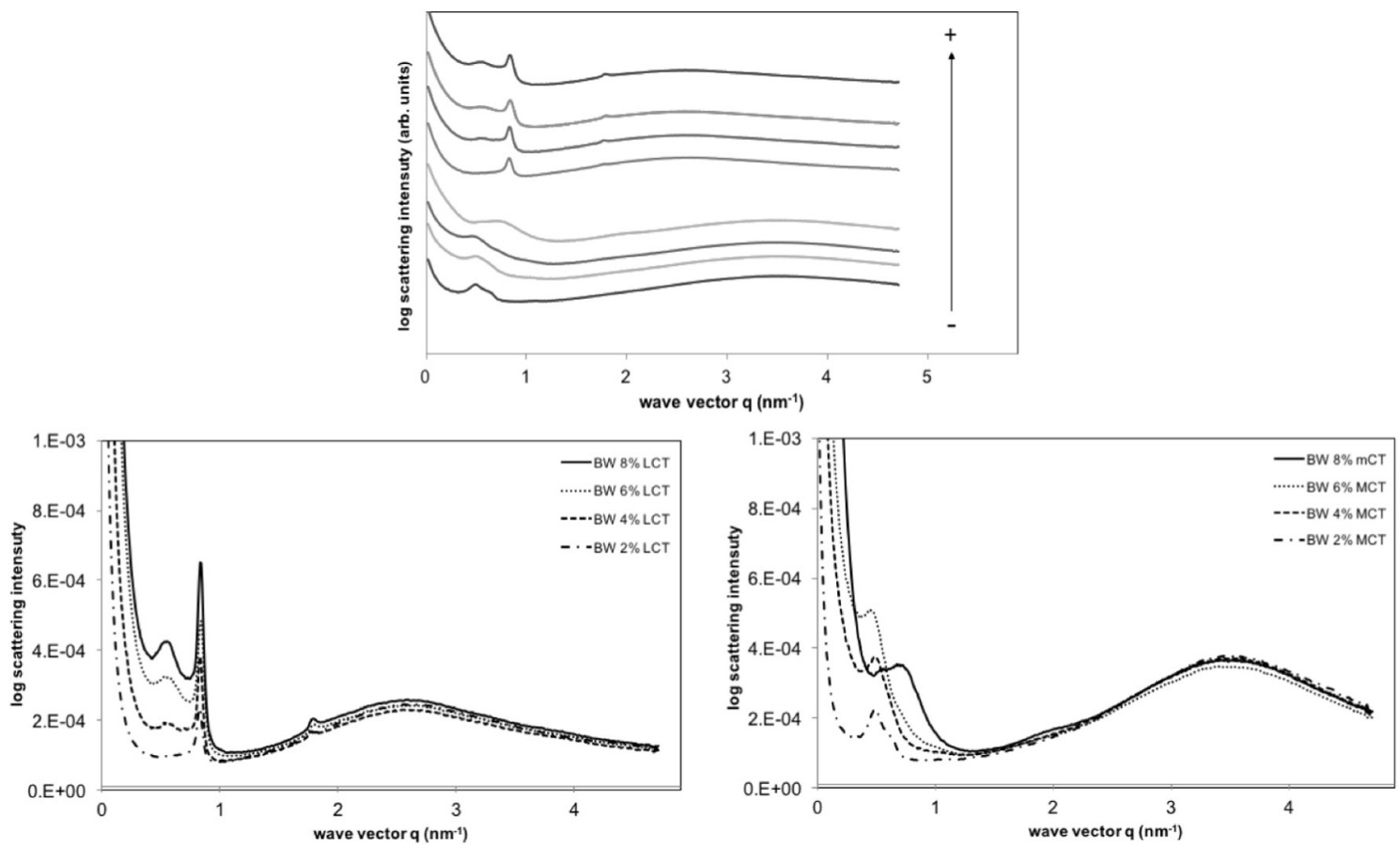

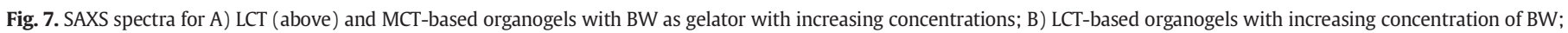
C) MCT-based organogels with increasing concentration of BW. Data regarding $1 \%$ of BW is not presented. 

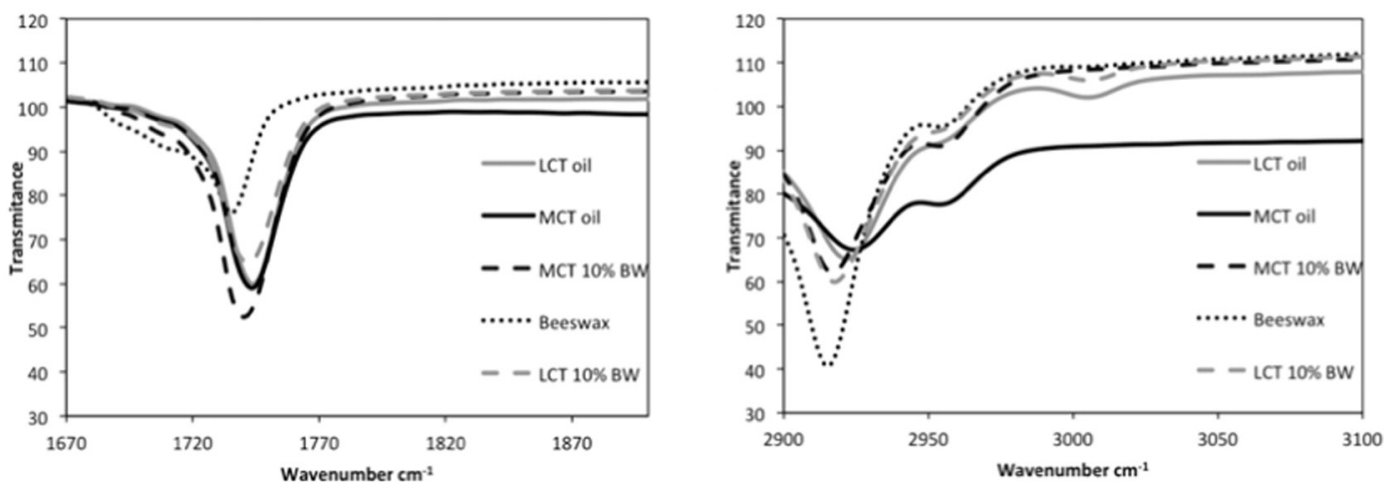

Fig. 8. FTIR spectra for LCT oil, MCT oil, beeswax and selected organogels samples.

interactions are occurring between the components (Yılmaz \& Ögütcü, 2014).

\section{Conclusions}

All organogels showed an increase of rheological properties for higher concentrations of gelator, justified by the effect of the more structured network formed when greater gelator concentrations are added to the system. Produced organogels revealed important features such as thermo-reversibility and thixotropy. This is an important exploration concerning the development of oil-based soft matter for food structuring applications.

No significant differences were observed regarding the isothermal rheological properties for the different types of organogels, but LCTbased organogels were stronger than MCT-based organogels during network formation (non-isothermal rheology). Regarding the difference of carbon chain of the oil phase, LCT-based organogels presented different spacing and placement between crystals, in a lamellar conformation, which was also observed was in non-isothermal rheological measurements. Because in this work the focus was structuring different oil phases (MCT and LCT), this effort allowed us to understand the impact of different carbon-chain oil phases and gelator concentrations in organogels' development stages. This study is very significant for the successful development of a feasible fat substitute for trans and saturated fats without compromising food structure and consumer perception, while providing added value to the final product.

\section{Acknowledgements}

The authors thank the Brazilian Synchrotron Light Laboratory (LNLS, Campinas, SP, Brazil) for the opportunity to carry out SAXS measurements. The author Miguel A. Cerqueira is recipient of a fellowship (SFRH/BPD/72753/2010) supported by Fundação para a Ciência e a Tecnologia, POPH-QREN and FSE (FCT, Portugal). The financial support of CAPES/FCT Project "Nanotechnological systems based in biocompatible ingredients: characterization, controlled release and in vitro digestion" (CAPES/FCT no. 348/13) and CNPq (Universal 479459/2012-6) are gratefully acknowledged. This study was supported by the Portuguese Foundation for Science and Technology (FCT) under the scope of the strategic funding of UID/BIO/04469/2013 unit and COMPETE 2020 (POCI-01-0145-FEDER-006684) and the Project RECI/BBB-EBI/ 0179/2012 (FCOMP-01-0124-FEDER-027462).

\section{References}

Acevedo, N. C., Peyronel, F., \& Marangoni, A. G. (2011). Nanoscale structure intercrystalline interactions in fat crystal networks. Current Opinion in Colloid \& Interface Science, 16(5), 374-383. http://dx.doi.org/10.1016/j.cocis.2011.05.004.
Amin, S., Barnett, G. V., Pathak, J. A., Roberts, C. J., \& Sarangapani, P. S. (2014). Protein aggregation, particle formation, characterization \& rheology. Current Opinion in Colloid E' Interface Science, 19(5), 438-449. http://dx.doi.org/10.1016/j.cocis.2014.10.002.

Barnes, H. A., Hutton, J. F., \& Walters, K. (1989). An introduction to rheology. Elsevier.

Behera, B., Singh, V. K., Kulanthaivel, S., Bhattacharya, M. K., Paramanik, K., Banerjee, I., \& Pal, K. (2015). Physical and mechanical properties of sunflower oil and synthetic polymers based bigels for the delivery of nitroimidazole antibiotic - A therapeutic approach for controlled drug delivery. European Polymer Journal, 64, 253-264. http://dx.doi.org/10.1016/j.eurpolymj.2015.01.018.

Blake, A. I., \& Marangoni, A. G. (2015). Plant wax crystals display platelet-like morphology. Food Structure, 3, 30-34. http://dx.doi.org/10.1016/j.foostr.2015.01.001.

Blake, A. I., Co, E. D., \& Marangoni, A. G. (2014). Structure and physical properties of plant wax crystal networks and their relationship to oil binding capacity. Journal of the American Oil Chemists' Society, 91(6), 885-903 doi: 10.1007/s11746-014-2435-0.

Cichero, J. A. Y., \& Murdoch, B. E. (2006). Dysphagia: Foundation. Theory and practice. Wiley.

Dassanayake, L. S. K., Kodali, D. R., \& Ueno, S. (2011). Formation of oleogels based on edible lipid materials. Current Opinion in Colloid E' Interface Science, 16(5), 432-439. http://dx.doi.org/10.1016/j.cocis.2011.05.005.

Dassanayake, L. S. K., Kodali, D. R., Ueno, S., \& Sato, K. (2009). Physical properties of rice bran wax in bulk and organogels. Journal of the American Oil Chemists' Society, 86(12), 1163-1173 doi: 10.1007/s11746-009-1464-6.

Dassanayake, L. S. K., Kodali, D. R., Ueno, S., \& Sato, K. (2012). Crystallization kinetics of organogels prepared by rice bran wax and vegetable oils. Journal of Oleo Science, 61(1), 1-9 doi: 10.5650/jos.61.1.

Duffy, N., Blonk, H. G., Beindorff, C., Cazade, M., Bot, A., \& Duchateau, G. M. J. E. (2009). Organogel-based emulsion systems, micro-structural features and impact on in vitro digestion. Journal of the American Oil Chemists' Society, 86(8), 733-741 doi: 10. 1007/s11746-009-1405-4.

Jang A. Bae, W. Hwang . H. S., Lee, H. G., \& Lee, S. (2015). Evaluation of canola oil oleogels with candelilla wax as an alternative to shortening in baked goods. Food Chemistry, 187, 525-529. http://dx.doi.org/10.1016/j.foodchem.2015.04.110.

Koh, S. -P., Arifin, N., Tan, C. -P., Yusoff, M. S. A., Long, K. Idris, N. A., \& Lai, O. -M. (2008). Rheological properties, oxidative stability and sensory evaluation of enzymatically synthesized medium- and long-chain triacylglycerol-based salad dressings. European Journal of Lipid Science and Technology, 110(12), 1116-1126 doi: 10.1002/ ejlt.200800066.

Konarev, P. V., Volkov, V. V., Sokolova, A. V., Koch, M. H. J., \& Svergun, D. I. (2003). PRIMUS: A Windows PC-based system for small-angle scattering data analysis. Journal of Applied Crystallography, 36(5), 1277-1282 doi: 10.1107/s0021889803012779.

Nugrahini, A. D., \& Soerawidjaja, T. H. (2015). Directed interesterification of coconut oil to produce structured lipid. Agriculture and Agricultural Science Procedia, 3, 248-254. http://dx.doi.org/10.1016/j.aaspro.2015.01.048.

Öğütcü, M., Arifoğlu, N., \& Yılmaz, E. (2015a). Storage stability of cod liver oil organogels formed with beeswax and carnauba wax. International Journal of Food Science and Technology, 50.(2), 404-412 doi: 10.1111/ijfs.12612.

Öğütcü, M., Yılmaz, E., \& Güneșer, O. (2015b). Influence of storage on physicochemical and volatile features of enriched and aromatized wax organogels. (journal article) Journal of the American Oil Chemists' Society, 92(10), 1429-1443 doi: 10.1007/ s11746-015-2719-z.

Patel, A. R., Schatteman, D. De Vos, W. H., Lesaffer, A., \& Dewettinck, K. (2013). Preparation and rheological characterization of shellac oleogels and oleogel-based emulsions. Journal of Colloid and Interface Science, 411, 114-121. http://dx.doi.org/10.1016/j.jcis 2013.08.039.

Pérez-Monterroza, E. J., Márquez-Cardozo, C. J., \& Ciro-Velásquez, H. J. (2014). Rheological behavior of avocado (Persea americana Mill, cv. Hass) oleogels considering the combined effect of structuring agents. LWT-Food Science and Technology, 59(2), 673-679. http://dx.doi.org/10.1016/j.lwt.2014.07.020.

Rocha, J. C. B., Lopes, J. D., Mascarenhas, M. C. N., Arellano, D. B., Guerreiro, L. M. R., \& da Cunha, R. L. (2013). Thermal and rheological properties of organogels formed by sugarcane or candelilla wax in soybean oil. Foodservice Research International, 50(1) 318-323. http://dx.doi.org/10.1016/j.foodres.2012.10.043.

Rogers, M. A. (2009). Novel structuring strategies for unsaturated fats - Meeting the zerotrans, zero-saturated fat challenge: A review. Foodservice Research International, 42(7) 747-753. http://dx.doi.org/10.1016/j.foodres.2009.02.024. 
Sawalha, H., Margry, G., den Adel, R., Venema, P., Bot, A., Flöter, E., \& van der Linden, E. (2013). The influence of the type of oil phase on the self-assembly process of $\gamma$ oryzanol $+\beta$-sitosterol tubules in organogel systems. European Journal of Lipid Science and Technology, 115(3), 295-300 doi: 10.1002/ejlt.201100395.

Toro-Vazquez, J. F., Morales-Rueda, J. A., Dibildox-Alvarado, E., Charó-Alonso, M., AlonzoMacias, M., \& González-Chávez, M. M. (2007). Thermal and textural properties of organogels developed by candelilla wax in safflower oil. Journal of the American Oi Chemists' Society, 84(11), 989-1000 doi: 10.1007/s11746-007-1139-0.

Vlachos, N., Skopelitis, Y., Psaroudaki, M., Konstantinidou, V., Chatzilazarou, A., \& Tegou, E. (2006). Applications of Fourier transform-infrared spectroscopy to edible oils. Analytica Chimica Acta, 573-574, 459-465. http://dx.doi.org/10.1016/j.aca.2006.05.034.

Yilmaz, F., \& Dagdemir, E. (2012). The effects of beeswax coating on quality of Kashar cheese during ripening. International Journal of Food Science and Technology, 47(12), 2582-2589 doi: 10.1111/j.1365-2621.2012.03137.x.

Yılmaz, E., \& Öğütcü, M. (2014). Properties and stability of hazelnut oil organogels with beeswax and monoglyceride. Journal of the American Oil Chemists' Society, 91(6), 1007-1017 doi: 10.1007/s11746-014-2434-1.
Yilmaz, E., Ogutcu, M., \& Yuceer, Y. K. (2015). Physical properties, volatiles compositions and sensory descriptions of the aromatized hazelnut oil-wax organogels. Journal of Food Science, 80(9), S2035-S2044 doi: 10.1111/1750-3841.12992.

Yılmaz, E., Öğütcü, M., \& Arifoglu, N. (2015a). Assessment of thermal and textural characteristics and consumer preferences of lemon and strawberry flavored fish oil organogels. Journal of Oleo Science, 64(10), 1049-1056 doi: 10.5650/jos.ess15113.

Yılmaz, E., Öğütcü, M., \& Yüceer, Y. K. (2015b). Physical properties, volatiles compositions and sensory descriptions of the aromatized hazelnut oil-wax organogels. Journal of Food Science, 80(9), S2035-S2044 doi: 10.1111/1750-3841.12992.

Zárubová, M., Filip, V., Kšandová, L., Šmidrkal, J., \& Piska, I. (2010). Rheological and crystalline properties of trans-free model fat blends as affected by the length of fatty acid chains. Journal of Food Engineering, 99(4), 459-464. http://dx.doi.org/10.1016/j. jfoodeng.2008.08.030. 